\title{
Grazing trajectory statistics and visualization platform based on cloud GIS
}

\author{
Dong Li, ${ }^{1,2}$, Chuanjian Wang ${ }^{2,3^{*}}$ (D), Qilei Wang ${ }^{1}$, Tianying Yan ${ }^{1}$, Wanlong Bing ${ }^{1}$ and Ju Wang ${ }^{1}$
}

\begin{abstract}
It is very important for ranchers and grassland livestock management departments to master the information on the trajectory and feeding behavior of the herd timely and accurately. Therefore, this study developed a statistics and visualization platform for grazing trajectory. The platform was implemented by using the Web AppBuilder for ArcGIS framework and ArcGIS Online server. In particular, the trajectory processing service on the server was used to calculate walking speed, walking trajectory and feed intake of the herd in the platform. And these results were published to the ArcGIS Online server. The relevant information was analyzed and displayed by Web AppBuilder for ArcGIS calling the data on ArcGIS Online. Moreover, the paltform provided some visualization functions to support the visualization of user-defined analysis results. When users use the functions of spatial analysis (such as buffer analysis, finding hot pots analysis and interpolation point analysis), they can choose to analyze spatial data and related field information to conduct customized spatial data analysis. In a short, the platform realized the visualization functions of feed intake statistics, walking speed statistics, spatial analysis, line chart analysis and pie chart analysis of spatial data related attributes. It can provide technical support and data support for the relevant management departments to monitor grazing information and study the living habits of the herd.
\end{abstract}

Keywords: GIS, Trajectory processing, Statistics, Visualization

\section{Introduction}

\section{Background}

Animal husbandry is an important part of agriculture. It has a very important impact on people's life and socioeconomic development [1]. Grassland is an extremely important natural resource in the development of animal husbandry [2]. Rational use of grassland resources is related to the sustainable development of animal husbandry.

However, most grassland has different degrees of grassland degradation [3]. Grassland degradation refers to the process of grassland ecosystem retrograde succession and productivity decline under unreasonable use. The phenomenon of grassland degradation is because humans continuously take a lot of material and energy from the grassland. The production speed of grassland is lower

\footnotetext{
*Correspondence: wcj\protect_si@ahu.edu.cn

${ }^{2}$ Geospatial Information Engineering Research Center, Xinjiang Production and Construction Corps, North Four Road, 832003 Shihezi, China

${ }^{3}$ School of Internet, Anhui University, Feixi Road, 230039 Hefei, China

Full list of author information is available at the end of the article
}

than the consumption speed for a long time, which violates the basic principle of ecological balance. The most important reason of grassland degradation is overgrazing. The herd takes much material and energy from the grassland. That is, the feed intake of the herd exceeds the tolerance limit of the grassland in the region [4-6].It breaks the ecological balance of grassland and leads to the failure of regeneration of grassland resources. This will endanger the development of animal husbandry.

In recent years, in order to prevent and solve grassland degradation, many countries and regions have formulated different plans based on the local grassland conditions. In particular, artificial grass planting is the most direct and efficient, but also the most costly solution. In contrast, it is the most practical and feasible to reduce grassland pressure and maintain the balance of grassland and livestock on the original grassland.

The unbalanced use of grassland will affect the measurement index of grass-livestock balance. Within a certain 
space, the terrain of local grassland is rugged, and the herd is not easy to feed. This makes the grassland of this area unused or lightly used, which wastes greatly grassland resources. However, overuse of grassland occurs in areas with flat terrain and easy feeding. It results in grassland degradation. Therefore, it is necessary to analyze the feeding behavior of the herd in time and accurately, which is of great significance to adjust grazing strategies, improve grassland productivity and protect grassland ecosystem $[7,8]$.

\section{Motivation}

Traditionally, feeding behaviors of the herd are mainly studied by animal husbandry technicians using observation method. They used counters, stopwatches, telescopes and other devices to track feeding behaviors of the herd, such as the walking speed and walking trajectory. They recorded and analyzed the time and number of feeding behaviors at fixed points [9-11]. Unfortunately, due to the limitations of people's number, devices' capabilities and research area, the information collected by this method is less. And this method is time-consuming, labor-intensive and poor timeliness. It is difficult to describe the spatiotemporal information of herd's feeding behavior intuitively and accurately [12]. Thus, it is difficult for ranchers and grassland livestock management departments to grasp feed rules and feed situations of the herd and make timely decisions on grassland improvement. Therefore, it is particularly important to develop a grazing trajectory statistics and visualization platform. The platform can process and analyze trajectory data of the herd. It can provide intuitive information on the herd's feeding behaviors for ranchers and grassland livestock management departments, so as to balance the use of grassland resources and promote the sustainable development of grassland livestock.

\section{Paper contributions}

The major contributions of this paper are summarized as follows.

1. The platform realizes the calculation of walking speed, walking trajectory and feed intake of the herd by using the GIS spatial analysis method.

2. This platform realizes the evaluation of feed situation of the herd and shows the proportion of different feed situation in the form of pie chart by using the grazing situation evaluation model.

3. In order to enable users to perform some corresponding trajectory analysis operations according to their own needs, the platform realizes the functions of buffer analysis, finding hot spots, interpolation point analysis and related trajectory query.

4. Conduct extensive experimental evaluations to demonstrate the efficiency and effectiveness of the platform.
The remainder of this paper is organized as follows. "Introduction" section provides an introduction to the outstanding problems of grassland livestock, especially the damage to the ecological environment caused by unreasonable feeding behaviors. "Related work" section focuses on related studies that investigate of feeding behaviors of the herd. "Methods" section describes the architecture of the platform and the principle of related core functions. Results section describes the results of the platform implementation and application cases. "Discussion and conclusions" section discusses the platform and concludes remarks and future directions.

\section{Related work}

In recent years, with the realization of modern animal husbandry informatization, many techniques have matured gradually, such as GPS technology, GIS technology, modern communication and computer technology. These techniques have been successfully applied on the field of grassland and animal livestock $[13,14]$ and produce much better results than the traditional ways, since they are not limited by conditions of time and terrain. They can record the grazing trajectory of the herd and generate spatiotemporal trajectory data containing the movement information of the herd. Then, the habits of the herd can be studied through trajectory data $[15,16]$. Monitoring sheep by GPS can evaluate the information of animal behaviors and the use of plant resources, as well as interactions with natural ecosystems. Therefore, it has become a common method in many countries [17].

In terms of feeding behaviors of the herd, many researchers have done the following research work. Arnon et al. [18] conducted his experiment at a study site in the semi-arid region of the Negev, northern Israel. They used GPS devices to record the trajectory of the herd. They analyzed the herd's walking speed by the factors of the pasture (slope, aspect, and distance between the herd and the corral). The speed of flock movement is greatly affected by the distance of the corral. The flock walks fastest when the distance between the flock and the corral is the largest or the smallest. As the slope increases, the walking speed of the flock decreases. The walking speed of the flock is also affected by the aspect. Henkin et al. [19] studied the effects of topographical factors on cattle foraging behavior in hilly areas. In this study, they used the LOTEK 2200 series GPS collar (Lotek Engineering, Newmarket, ON, Canada) to record the trajectory of the herd at intervals of $5 \mathrm{~min}$ utes. They analyzed the grazing trajectory under different grazing intensities and different seasons. They found that the herd likes to eat in flat terrain. Only when the grazing intensity tends to be saturated or the forage biomass is low will it eat in areas with steep terrain. Pérez et al. [20] used global positioning system and GPRS to track and monitor free-range cow. The circadian rhythm map of the 
walking distance of cattle in a few hours was obtained. It was concluded that the average daily walking distance of cattle is 3.15 kilometers. Mysterud et al. [21] used GPS devices to record the movement trajectory of sheep. The conclusion showed that in the area with high vegetation coverage, sheep selection increased in areas with an overall higher productivity. Liao et al. [22] used GPS collars to record the feeding behavior of the herd. The relationship between the behavior types of cattle and the statistical parameters of their movement was established. Then, the distribution of time and space of their movement behavior and resource selection was analyzed predicted. Wang et al. [12] used GPS devices to obtain temporal and spatial trajectory data of the herd. The feed intake distribution of the herd was obtained by buffer zone and grid analysis. Akasbi et al. [23] Kawamura et al. [24] used GPS devices to obtain temporal and spatial trajectory data of the herd. The feed intake distribution of the herd was obtained by grid method. The above researchers mainly analyzed the feeding behavior and habits of the herd by the recorded trajectory data. However, few research results were applied to the corresponding grazing management platform to show visually.

In terms of developing systems, many researchers have done the following research work. Chen [25] developed a satellite grazing system based on GPS and GIS by WebGIS, GNSS, mobile communication and other techniques. The functions of herd position display, motion trajectory query and virtual fence were realized in the system. Cheng [26] developed a system by GPS positioning and GPRS data transmission technology. The functions of herd position display and track query were realized in the system. Du et al. [15] proposed a new method of dividing and clustering based on the disturbance of trajectory speed. Firstly, the trajectory of the herd was divided by speed's threshold. Then, the divided trajectory data was clustered to identify different living areas of livestock. Finally, in order to show the different intensity of each region, the kernel density analysis method was used to visualize the clustering regions of different trajectories. In the above research work, GPS positioning technology was applied to the monitoring of the herd. The informatization of animal husbandry was realized in a certain extent. However, there are few functions about the change rule, evaluation and prediction of the feed intake of the herd. It can not meet the higher demands of grassland animal husbandry for herd's monitoring.

Spatial data has three basic features: spatial feature, attribute feature and time feature. In the era of big data, spatial data processing, analysis and visualization have the characteristics of large data volume and complicated calculation compared with other data. Spatial data needs to be processed and analyzed in time, so it has a higher demand for transmission speed, computing power, Internet and IOT deveices. Nowadays, with the rapid development of 5G network and the continuous improvement of IOT, there is a good foundation for the development of grazing trajectory statistics and visualization platform. With the continuous improvement of service quality in the cloud field and the Internet, cloud computing can solve the problem of insufficient computing power better. At the same time, favorable conditions can be provided for the transmission of spatial data with the rapid development of network technology [27-30]. The Internet and the IOT have focused on edge computing [31-33]. The lack of computing power of ECS was alleviated by IOT devices [34]. Moreover, with the continuous deepening of related research, the reliability and availability of the system have also been improved greatly [35-38]. Therefore, the study has certain technical support to develop grazing tracjectory statistics and visualization platform.

\section{Methods}

\section{Function design of the platform}

The grazing tracjectory statistics and visualization platform has six functional modules: data management, map operation, information query, spatial analysis, statistical analysis, and print sharing. The functional modules of the platform are shown in Fig. 1.

Data management adopted SQL Server+ArcSDE spatial data engine to realize the functions of adding, deleting and querying of basic pasture data, herd trajectory data and device information data. The operation of the map mainly includes basic operations such as zoom, translation, and eagle eye. It also includes the replacement of the platform base map and the display and hiding of other layers. Information query is mainly divided into spatial query and attribute query. Spatial query includes both pasture

\section{Grazing trajectory statistics and visualization platform based on cloud GIS}
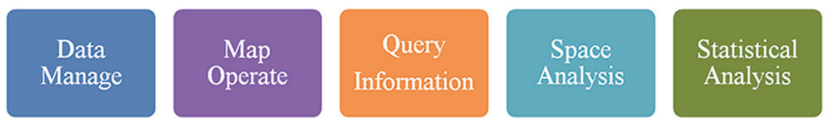

Print and

Share

Fig. 1 The functional modules of the platform 
location information query and herd trajectory information query. Attribute query can query the corresponding attribute information of herd trajectory points and trajectory segments. Spatial analysis includes buffer zone analysis, finding hot spots, and predictive analysis, etc. It is used to monitor and predict the feeding behaviors of the herd. Statistical analysis is mainly to analyze the walking speed, feed intake and feed situation of the herd. Then, corresponding line charts and pie charts are generated and displayed on the client. Print and share include the print and share of ranch basic maps and herd trajectory information thematic maps.

\section{Platform structure design}

This platform was developed by using browser and server structure ( $\mathrm{B} / \mathrm{S}$ structure). The architecture of the platform is composed of presentation layer, business logic layer and data layer, as shown in Fig. 2.

\section{Presentation layer}

The presentation layer used the Web AppBuilder for ArcGIS framework that is a application framework to build views. Web AppBuilder for ArcGIS is a crossplatform web app creation framework. It has the advantages of configurable and scalable. It can be used to build $2 \mathrm{D}$ and $3 \mathrm{D}$ web applications. A highly customized map is realized by creating quickly map appearances and widget templates without coding. The framework is tightly integrated with Portal/AGOL. The maps published on ArcGIS Online can be seamlessly added to the client display by the framework. The framework adopts responsive layout design. The created application can adapt to screens of various sizes by the framework. It can run on PCs, tablets, and smartphones of various types. This design structure makes the geographic information service simple and flexible. It also improves the reusability of modules and the schedulability of the overall configuration. This platform used ArcGIS API for JavaScript and Web AppBuilder for ArcGIS to carry out modular design and implementation. In order to make the interface rich and easy to operate, the platform put tools with similar logic together (such as map zoom, pan, panorama of the map operation group ; buffer analysis, finding hot spots and prediction analysis of the spatial analysis group), which enhances the operability of the interface.

\section{Business logic layer}

The business logic layer is divided into GIS services and Web services. GIS services include basic map services, trajectory processing services and other business services. The main function of the trajectory processing services is to extract the herd trajectory data from the Microsoft SQL Server2008 database, and use the relevant model to calculate the corresponding walking speed of the herd, walking trajectory, and feed intake of the herd. Then, publish the results to the ArcGIS Online server by basic map services and other business services. Finally, the results are displayed visually by Web AppBuilder for ArcGIS. ArcGIS Online is a cloud computing-based application development support platform. It includes global basemaps, map data, applications, configurable application templates, GIS tools and APIs. Therefore, it can be used to create Web maps, publish GIS services, share maps, share data and applications. It also can manage organizational content and multiple users. Web services are responsible for hosting web applications and services. Tomcat is used to

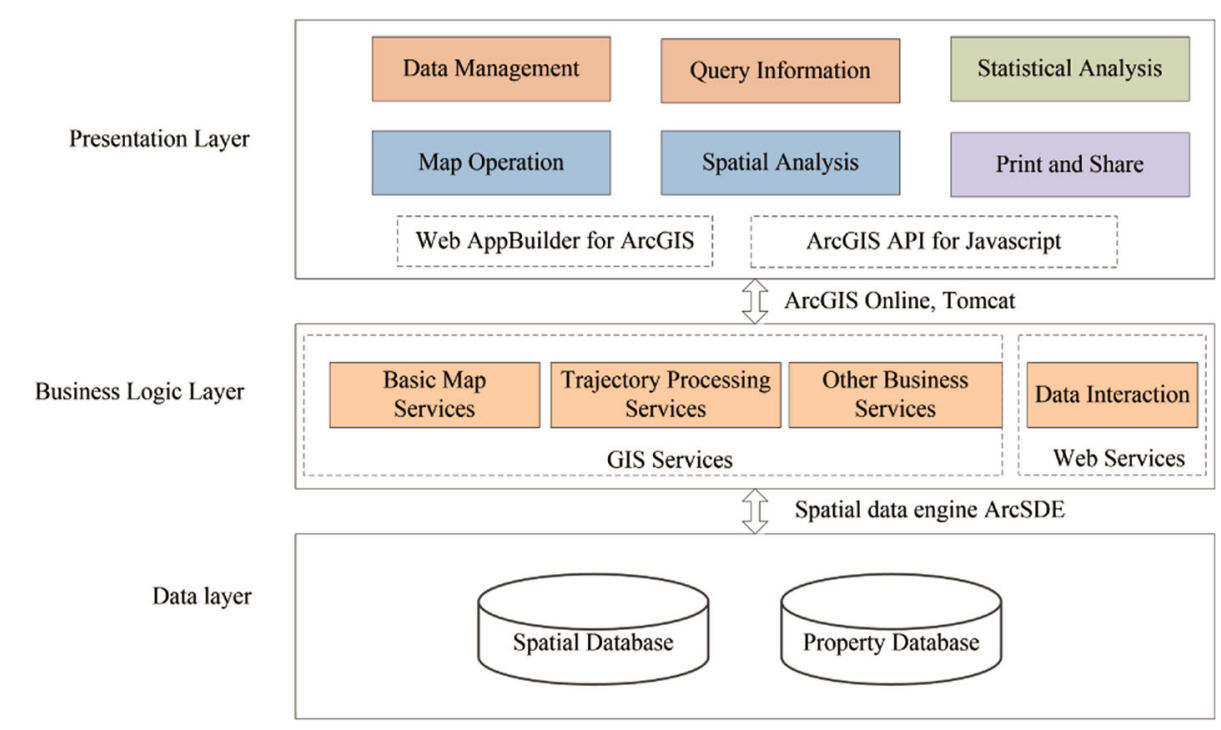

Fig. 2 The architecture diagram of the platform 
deploy web applications for Internet access. The web application or web service can respond to the client's request, and return the data to the client, so as to realize the data interaction between the client and the server.

\section{Data layer}

The data layer used SQL Server+ArcSDE spatial data engine to standardize and store the trajectory data (such as trajectory point data, trajectory segment data, herd feeding information data, etc.), vector data (such as pasture boundary data, herd boundary data, etc.), raster data (such as herd feeding grid data), and devices information data. The main task of this layer is to design and build a comprehensive database of spatial attributes. Firstly, determine the type and characteristics of all data. Then test, optimize and maintain the data. Finally, bulid a comprehensive database including basic boundary database, herd trajectory database, herd feeding information spacetime database, devices information database.

\section{Core models}

\section{Trajectory and speed model}

The grazing trajectory is a sequence of time and space points. It consists of a series of data points that change with time and position. The location information is denoted by the acquired latitude and longitude. $T=$ $\left\{T_{1,1} \cdots, T_{i, j}, \cdots, T_{n, m}\right\}, T_{i, j}=\left\{P_{1}, P_{2}, \cdots, P_{n}\right\}, P_{i}=$ $\left\{\right.$ Lat $\left._{i}, \operatorname{Lng}_{i}, t_{i}\right\}(0 \leq i \leq n, 0 \leq j \leq m)$. Where $T$ denotes the set of herd trajectories in a period of time. $T_{i, j}$ denotes the set of trajectory points of the $j$-th monitored sheep on the $i$-th day. It is a collection of spatial points with time series. $P_{i}$ denotes the $i$-th trajectory data points. $L a t_{i}$ denotes latitude value. $L n g_{i}$ denotes longitude value. $t_{i}$ denotes the positioning time.

Trajectory segment: Several local trajectories are obtained by segmenting the trajectory. The segmentation method is based on the GPS points contained in the trajectory segment. The GPS points are not less than 2 . The smaller the number of trajectory points contained in the trajectory segment, the closer the fitted grazing trajectory is to the real grazing trajectory. Therefore, the platform used a segmentation method that contains 2 trajectory points in the trajectory segment to divide the trajectory into several trajectory segments.

Grazing speed: The trajectory segment is a sequential trajectory displacement connected by two trajectory points(Fig. 3). Therefore, grazing speed $\overrightarrow{v_{i}}$ is the ratio of trajectory segment $\overrightarrow{P_{i} P_{i+1}}$ and the time difference $t_{i+1}-t_{i}$ (Eq. 1):

$$
\overrightarrow{v_{i}}=\frac{\overrightarrow{P_{i} P_{i+1}}}{t_{i+1}-t_{i}}
$$

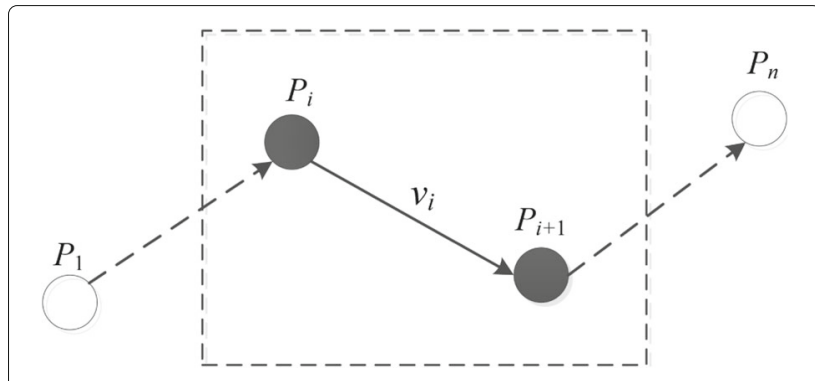

Fig. 3 The speed of grazing trajectory point in continuous time

\section{Calculation model of feed intake}

In the actual grazing process, the grazing trajectory of the herd shows a certain regularity. It presents the strip spatial distribution characteristics. The range of activity produced by the herd feeding is simulated by buffer processing of trajectory segment $L_{i}$, that is part grazing area, as shown in Fig. 4.

The feed time of the herd is continuous. Based on this feature, this study assumed that there was no significant difference in the feed intake per unit time and the total daily feed intake of the herd. The effective feed time $t_{i}$ of the herd is obtained from each trajectory segment $L_{i}$. The total daily feed intake is allocated to each trajectory segment $L_{i}$ according to the ratio of the effective feed time $t_{i}$ corresponding to the trajectory segment $L_{i}$ and the daily feed time $t_{A D F T}$. Finally, the feed intake $I_{i}$ of the PGA corresponding to the trajectory segment $L_{i}$ is obtained [12]:

$$
\begin{aligned}
& I_{i}=I_{D H F I} \cdot \frac{t_{i}}{t_{A D F T}}(0<\mathrm{i}<n) \\
& t_{A D F T}=\sum_{i=1}^{n-1} t_{i}=\sum_{i=1}^{n-1}\left(P_{i+1} t-P_{i} t\right)
\end{aligned}
$$

Where $I_{D H F I}$ denotes the daily feed intake of the herd. In this study, the experimental parameter of the $I_{D H F I}$ is $170 \times 2000 \mathrm{~g}, 170$ denotes that there are 170 sheep in the herd, and $2000 \mathrm{~g}$ denotes that the total daily feed intake of each sheep is $2000 \mathrm{~g}$. $t_{A D F T}$ denotes the all-day feed time of the herd. $P_{i+1} t$ denotes the positioning time of the $i+1$ th trajectory point $P_{i+1} . P_{i} t$ denotes the time of the $i$-th trajectory point $P_{i}$. The difference between them is the feeding time $t_{i}$ of the trajectory segment $L_{i}$. In order to calculate the feed intake of per unit area, the area $S_{i}$ of the part grazing corresponding to each trajectory segment $L_{i}$ is considered. Therefore, feed intake of per unit area of $L_{i}$ is:

$$
F_{i}=t_{i} \cdot \frac{I_{D H F I}}{\sum_{i=1}^{n-1} t_{i} \cdot S_{i}}=\left(P_{i+1} t-P_{i} t\right) \cdot \frac{I_{D H F I}}{\sum_{i=1}^{n-1}\left(P_{i+1} t-P_{i} t\right) \cdot S_{i}}
$$




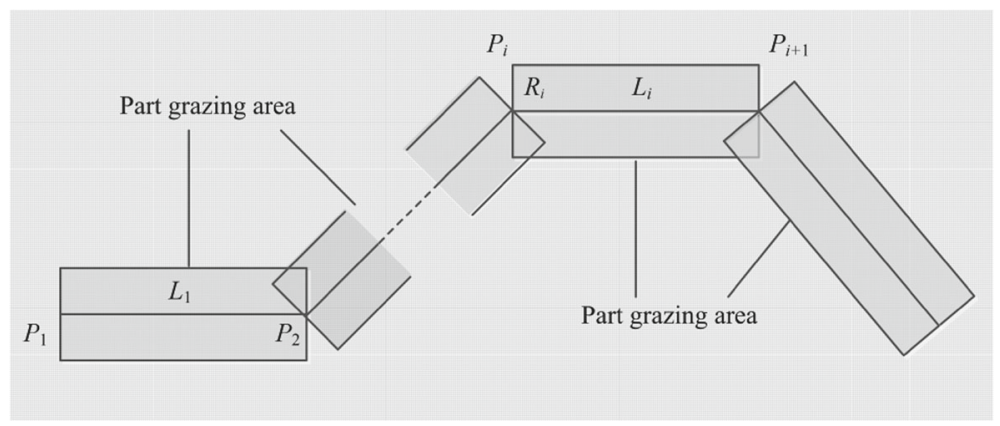

Fig. 4 Schematic diagram of part grazing area generation method

\section{Evaluation model of feed situation}

So far, there is no certain standard for the division of herd feed situation. In addition, it is difficult to find out the accurate classification standard for different types of herd feed in different regions. The boundary between the intervals will be too rigid, if it is divided strictly by the classification interval. However, jurisdiction degree theory of fuzzy mathematics can be used to quantitative evaluation. Fuzzy recognition can make a scientific, reasonable and practical quantitative evaluation for the fuzzy information. Therefore, this platform used a simple jurisdiction function based on the descending half trapezoid, the rising half trapezoid and the intermediate symmetry in fuzzy mathematics to evaluate the feed situation of the herd [39]. Taking the feed intake as the domain, the jurisdiction functions of the fuzzy comment set $\mathrm{V}=$ (good, moderate, poor) are given. The poor feed situation of the herd is divided into two different jurisdiction functions: one is light feed (Eq. 5) and the other is over feed (Eq. 6).

$$
\begin{aligned}
& \mu_{11}(x)= \begin{cases}1 & \left(x<b_{1}\right) \\
\frac{b_{2}-x}{b_{2}-b_{1}} & \left(b_{1} \leq x \leq b_{2}\right) \\
0 & \left(x>b_{2}\right)\end{cases} \\
& \mu_{12}(x)= \begin{cases}0 & \left(x<b_{4}\right) \\
\frac{-b_{4}+x}{b_{5}-b_{4}} & \left(b_{4} \leq x \leq b_{5}\right) \\
1 & \left(x>b_{5}\right)\end{cases}
\end{aligned}
$$

The intermediate symmetrical distribution function is used to estimate moderate feed and good feed of the herd (Eqs. 7, 8, and 9).

$$
\mu_{21}(x)= \begin{cases}\frac{x-b_{1}}{b_{2}-b_{1}} & \left(b_{1} \leq x \leq b_{2}\right) \\ \frac{b_{3}-x}{b_{3}-b_{2}} & \left(b_{2}<x \leq b_{3}\right) \\ 0 & \left(x<b_{1} \text { or } x>b_{3}\right)\end{cases}
$$

$$
\begin{gathered}
\mu_{22}(x)= \begin{cases}\frac{x-b_{3}}{b_{4}-b_{3}} & \left(b_{3} \leq x \leq b_{4}\right) \\
\frac{b_{5}-x}{b_{5}-b_{4}} & \left(b_{4}<x \leq b_{5}\right) \\
0 & \left(x<b_{3} \text { or } x>b_{5}\right)\end{cases} \\
\mu_{3}(x)= \begin{cases}\frac{x-b_{2}}{b_{3}-b_{2}} & \left(b_{2} \leq x \leq b_{3}\right) \\
\frac{b_{4}-x}{b_{4}-b_{3}} & \left(b_{3}<x \leq b_{4}\right) \\
0 & \left(x<b_{2} \text { or } x>b_{4}\right)\end{cases}
\end{gathered}
$$

Where $b_{i}$ is the boundary of different feed intake, $i=1,2$, ..., $5 ; x$ is the feed intake, $\mathrm{g} / \mathrm{m}^{2}$.

\section{Spatial analysis models}

The spatial analysis models used in this platform include buffer analysis model, finding hot pots model and interpolation point analysis model.

Buffer analysis model is one of the important spatial analysis functions of geographic information systems. It has a wide range of applications in transportation, forestry, resource management, and urban planning, such as the delimitation of the protected areas around lakes and rivers, the selection of automobile service areas, and the establishment of buffer areas far from the street network in residential areas, etc. Buffer analysis is based on point, line and face entities to build automatically a polygon layer of buffer in a certain width range, and then the superposition of this layer and the target layer is built to analyze and obtain the required results. The buffer based on point feature is usually a circle with a point as the center and a certain distance as the radius. The buffer based on line feature is usually a parallel strip polygon with the line as the central axis and a certain distance from the central axis. The buffer based on polygon boundary of the area feature is a new polygon, which can be generated by extending a certain distance outwards or inwards. The buffer program traverses each vertex of the input features and builds a 
buffer offset. The output buffer features are built by using these offsets.

Finding hot spots model is a spatial clustering which is used to identify high values (hot spots) or low values (cold spots) or data count with statistical significance in data. This model is used to find hot spots and cold spots of the herd feed information clustering. The map layer shows hot spots in red and cold spots in blue. The darkest red features represent the strongest clusters of high values or point density. The clusters associated with these features are not randomly generated results. They have $99 \%$ degree of confidence. Similarly, the darkest blue features are associated with the strongest low-value spatial clustering or the lowest point density. Beige features do not belong to statistically significant clusters. The spatial patterns associated with these features are most likely the results of random processes and random production.

Interpolation point analysis model can predict the values of new locations based on the values of a set of trajectory points. This model gets each point data with a value and returns the area classified by the predicted value. The point layer is used as the input layer. The input layer must have a numeric field to be the basis of interpolation. The interpolation speed, precision, and intermediate region can be optimized by setting the interpolation point model. The standard error layer can be created by selecting the output prediction error check box. The $95 \%$ confidence interval in the interpolation layer can be calculated by the interpolation value, the two standard upper bound errors and the two standard lower bound errors.

\section{Platform implementation}

This platform was developed by Web AppBuilder for ArcGIS framework, ArcGIS API for JavaScript and HTML5 technology. The paltform was a Web application with a beautiful user interface and rich common functions of GIS. ArcGIS Online was used as the GIS server and Tomcat7 was used as the Web server for the server of this platform. The database used Microsoft SQL Server 2008+ArcSDE spatial data engine to manage data. Based on Dojo development components, the platform was designed and built in REST style to enhance its scalability.

\section{Trajectory and speed}

The calculation method of trajectory and speed is shown in Fig. 5. Firstly, input the trajectory points of herd. Secondly, a point feature layer is made based on the trajectory points and sort it by sheep IDs and gpstime. Thirdly, make a tracking layer and calculate trajectory and walking speed of herd. Forthly, build a trajectory table. Fifthly, input sheep ID. The trajectory and speed of the sheep is obtained by querying the trajectory table.

\section{Feed intake}

The calculation method of feed intake is shown in Fig. 6 . The calculation steps of daily feed intake of herd are as follows. The first step: Input daily trajectory segments of herd and sheep ID. The trajectory segment of the sheep is obtained by querying the daily trajectory segments of herd. Input daily trajectory points of herd, and sort them according to sheep IDs and gpstime to get the sorted trajectory points. The second step: The distance between the trajectory points and the trajectory segment $L_{i}$ is calculated by using the calculation method from point to segment. The third step: The maximum distance $R_{i}$ is obtained by summarizing and calculation method. It is used as the buffer distance of the trajectory segment $L_{i}$. The fourth step: Build grazing buffer. The grazing buffer of each trajectory segment $L_{i}$ is built by translating the distance $R_{i}$ on both sides of the trajectory segment $L_{i}$ along the vertical direction. The fifth step: Calculate buffer area. The buffer area is calculated by the buffer distance $R_{i}$ and the length of the trajectory segment $L_{i}$. The sixth step: Input the formula of feed intake. Daily feed intake polygon data is obtained by calculating daily feed intake of herd.

For the calculation steps of multi-day feed intake are as follows. The first step: Input $n(n>1)$ daily feed intake polygon data. The $\mathrm{n}$ daily feed intake raster data is obtained by converting polygon data into raster data. Input pasture polygon data and convert its geographic coordinate system into projection coordinate system. The second step:

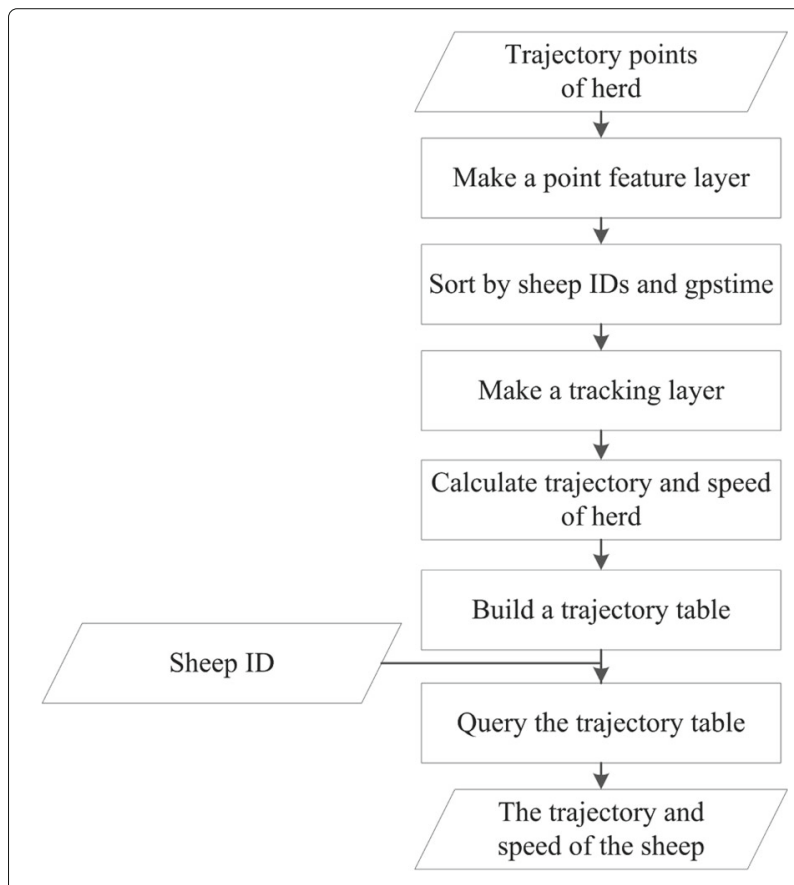

Fig. 5 Implementation flowchart of trajectory and speed model 

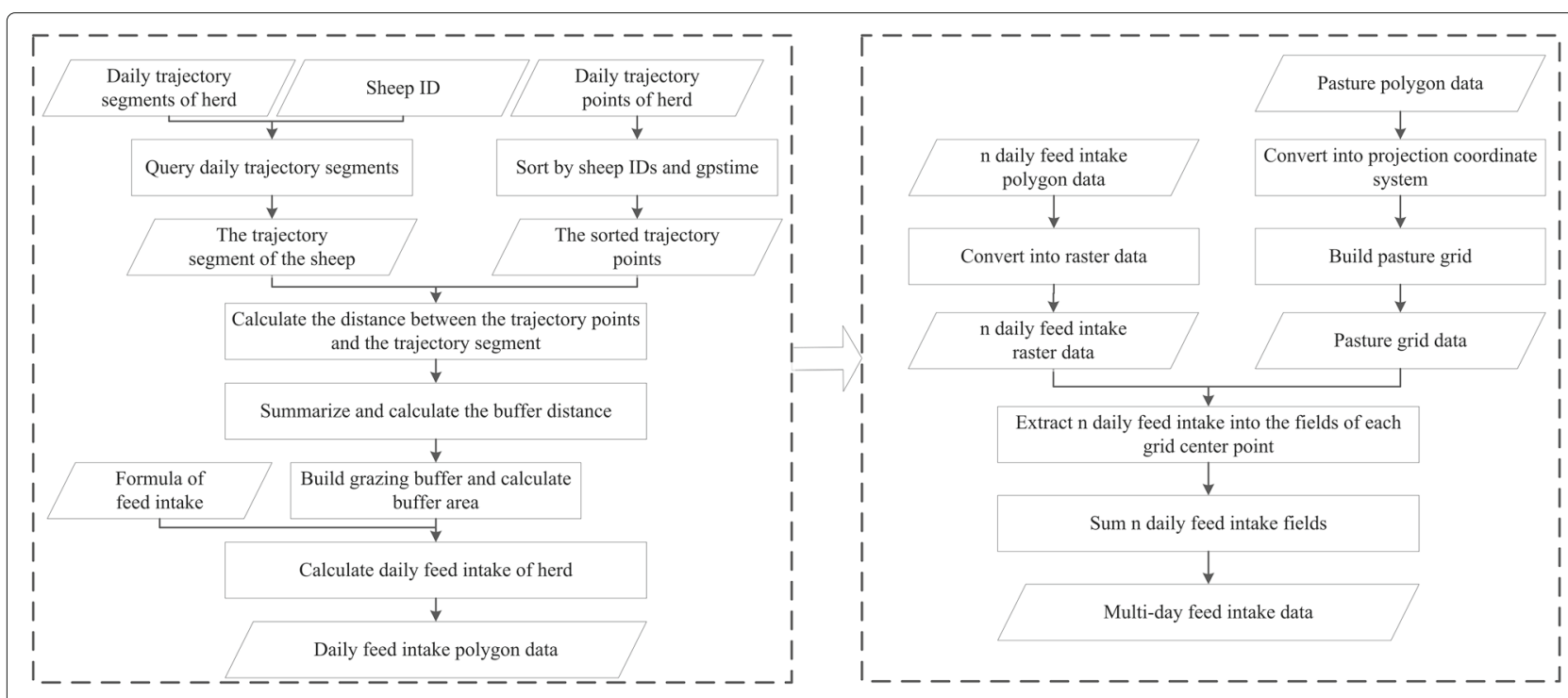

Fig. 6 Implementation flowchart of feed intake model

The pasture grid data is obtained by building pasture grid. The third step: Extract $\mathrm{n}$ daily feed intake into the fields of each grid center point. The forth step: The multi-day feed intake data is obtained by summing $\mathrm{n}$ daily feed intake fields.

\section{Feed situation}

The steps to evaluate the feed situation of the herd are as follows. Firstly, a new field needs to be added into the feed intake table to store the evaluation result of the feed situation. Secondly, the feed situation of the herd is evaluated by the evaluation model of feed situation. And, the result is stored in the new field. Finally, the feed situation of the herd is showed visually on the client by the pie chart of the platform. Therefore, users can grasp intuitively the feed situation of the herd in different areas. It is helpful for users to formulate feed plans reasonably and use grassland resource in a balanced manner. It also can promote the sustainable development of grassland livestock.

\section{Spatial anlysis}

The spatial analysis function relies on the Web AppBuilder for ArcGIS framework. The Analysis widget of the framework provides an easy way to use ArcGIS Online or ArcGIS Enterprise spatial analysis tools in application. The analysis widget provides 25 analysis tools. With 25 analysis tools, one tool or multiple tools can be configured in a widget. The platform used buffer analysis tool, finding hot spots tool and interpolation analysis tool of the analysis widget to realize the spatial analysis function. In order to ensure that the spatial analysis function is used normally, each user is given the following permissions:

1. Create, update, and delete content

2. Publish hosted features

3. Spatial analysis

\section{Results}

The platform used a dashboard theme, including a main view and an attribute table with drawer effects and five widgets. The main view displays the map and provides a series of map operation options, which include positioning, zooming the map, panning the map, returning to the center of the main map, selecting display layers, scale, print and share. Five widgets analyzes spatial data from different angles.

In this study, the Ziniquan pasture of Shihezi City, Xinjiang production and construction corps was taken as an example to test the application of the platform. The herd trajectory data in the study area from July 28 to July 31 , 2016 was processed and visualized, and the results were shown in Fig. 7. Figure 7 was the main view of the platform. It showed the range of the pasture and the trajectory points of the herd. The pasture range data and the trajectory point data of the herd can be freely selected by the attribute table. And a series of condition filtering queries can be conducted by the attribute table, as shown in Fig. 8 . According to the query data, the feeding behavior of the herd can be deeply explored.

The first widget of this platform shows the total feed intake of the herd during this period.

The second widget provides spatial analysis services, including buffer analysis service, finding hot spots service and interpolation point analysis service. Buffer analysis service is mainly applicable to small-scale pastures. According to the gathering and survival characteristics of the herd, the range of the herd activities can be inferred from the trajectories of some sheep. Taking the sheep with device ID 6021265 as an example, the range of the herd activities was obtained, as shown in Fig. 9. Finding hot spots service is the main spatial analysis service. 


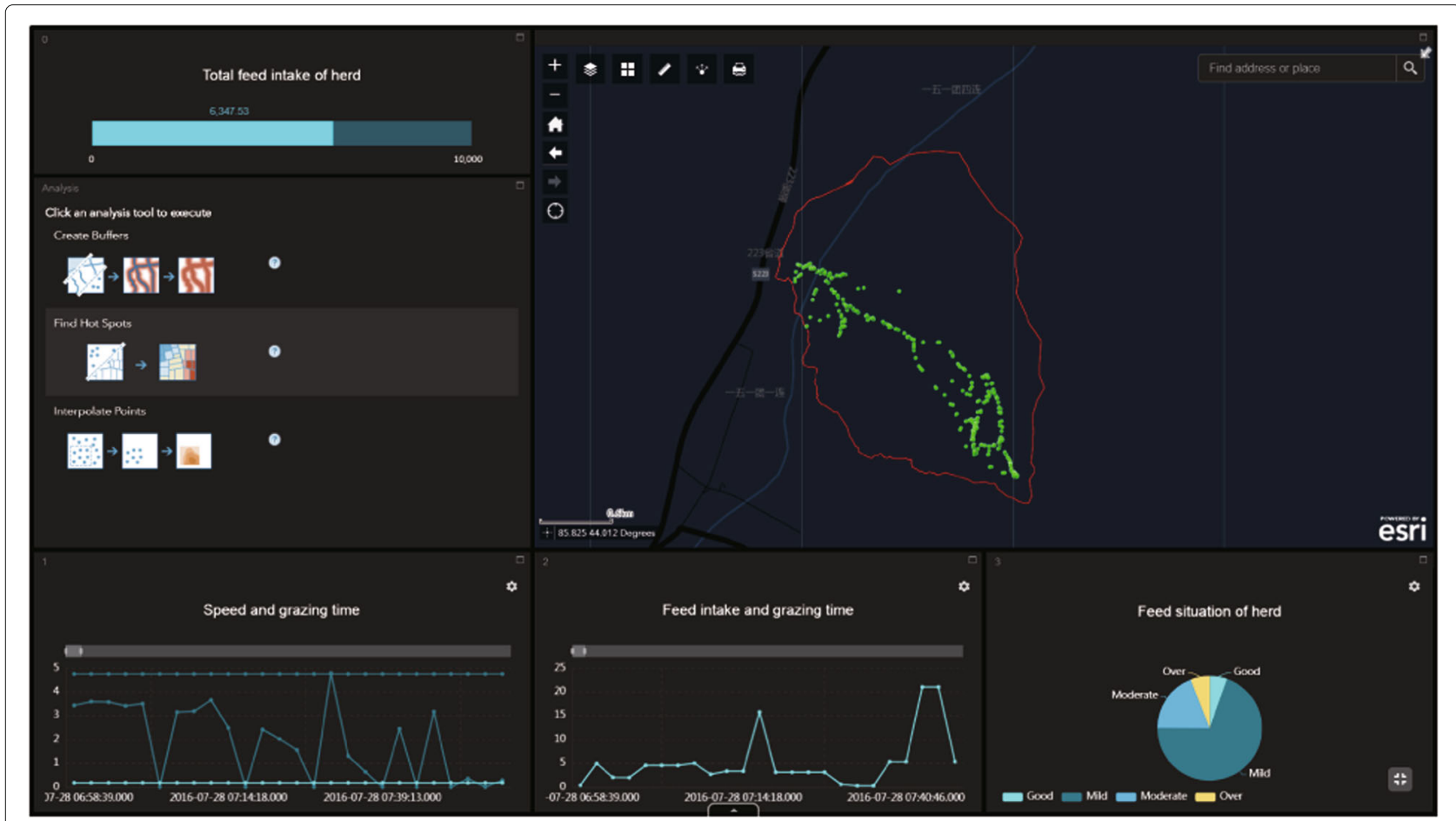

Fig. 7 The main view of the platform

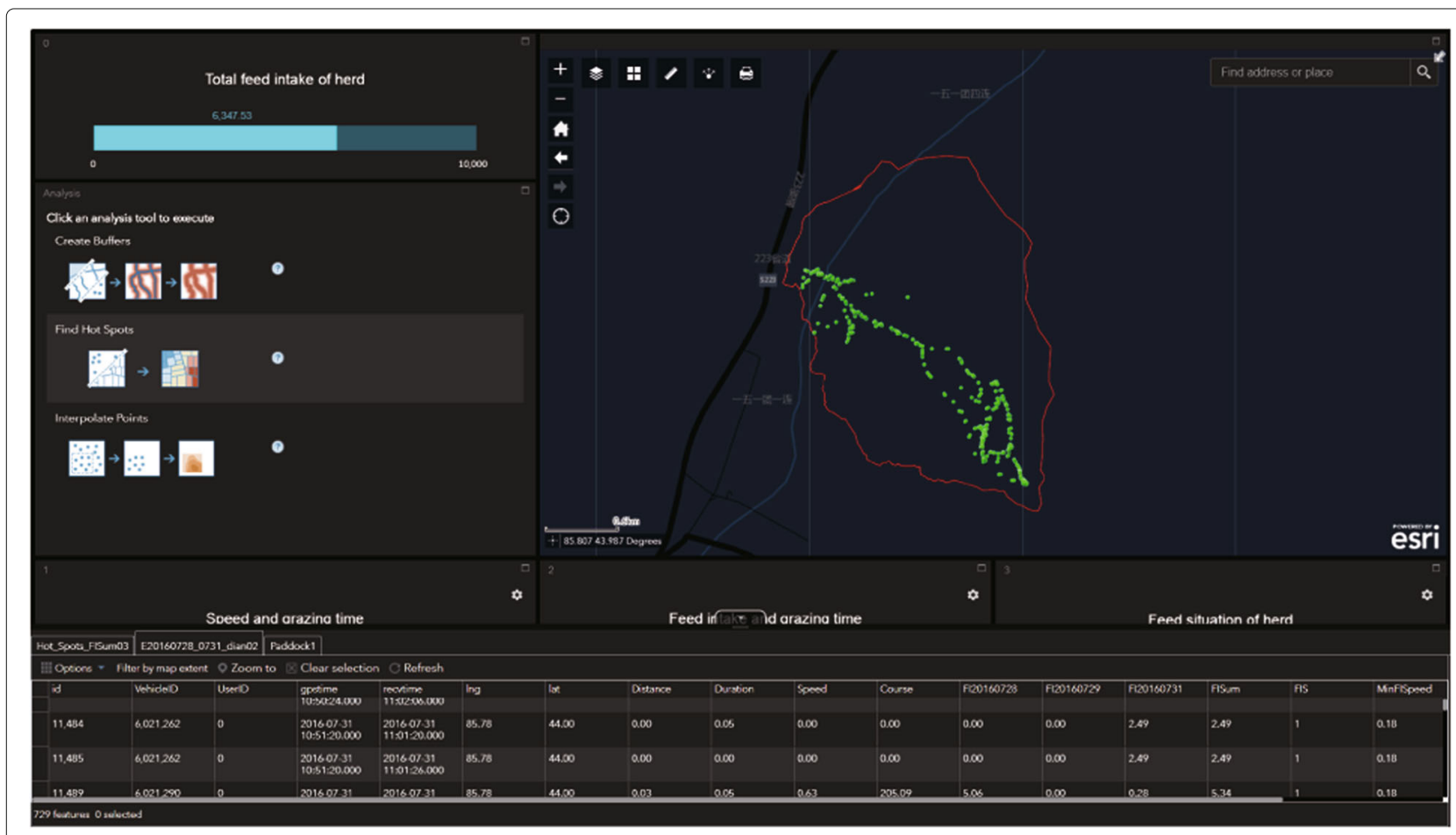

Fig. 8 The attribute table of the platform 


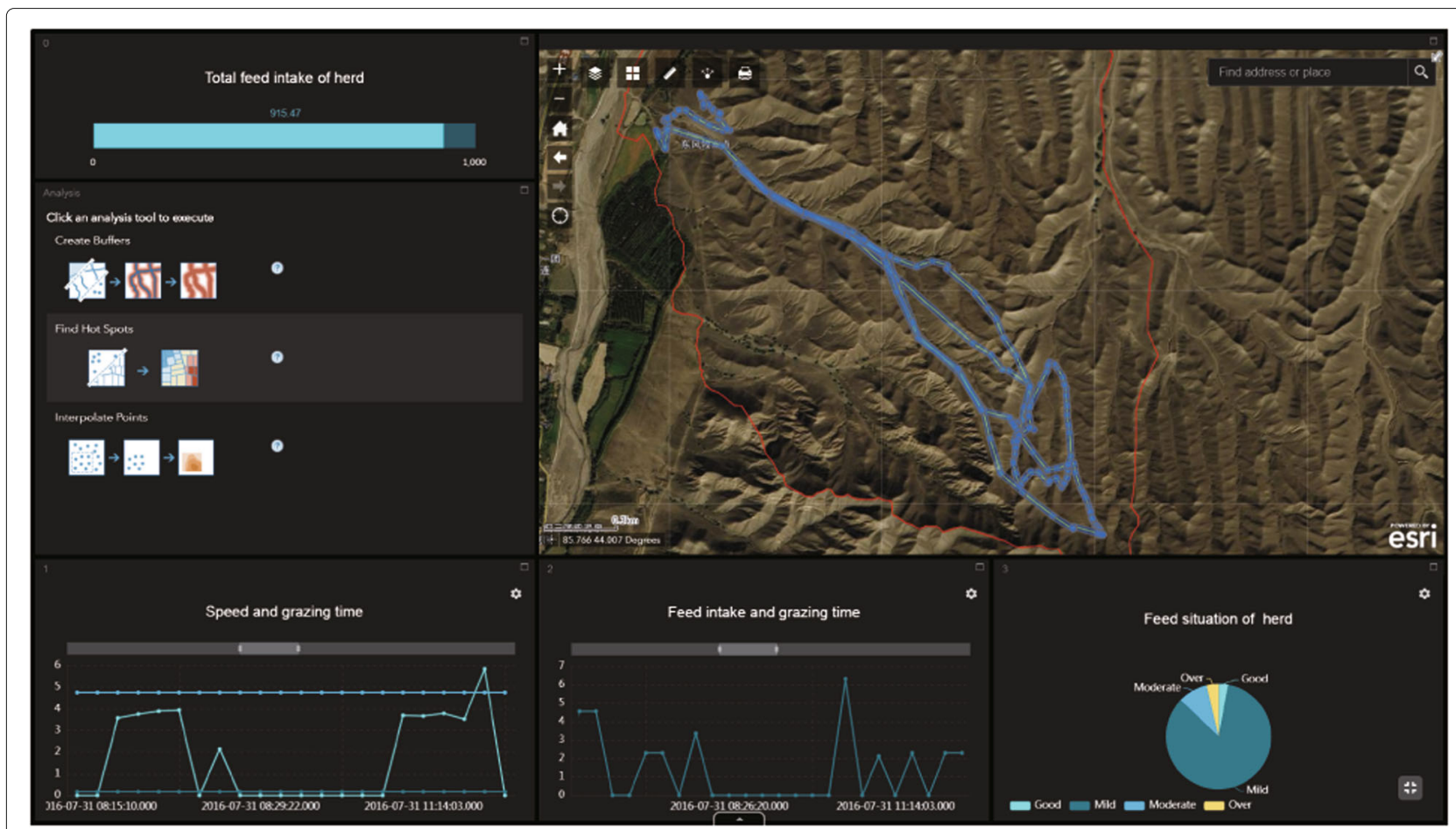

Fig. 9 The buffer analysis result diagram of the platform

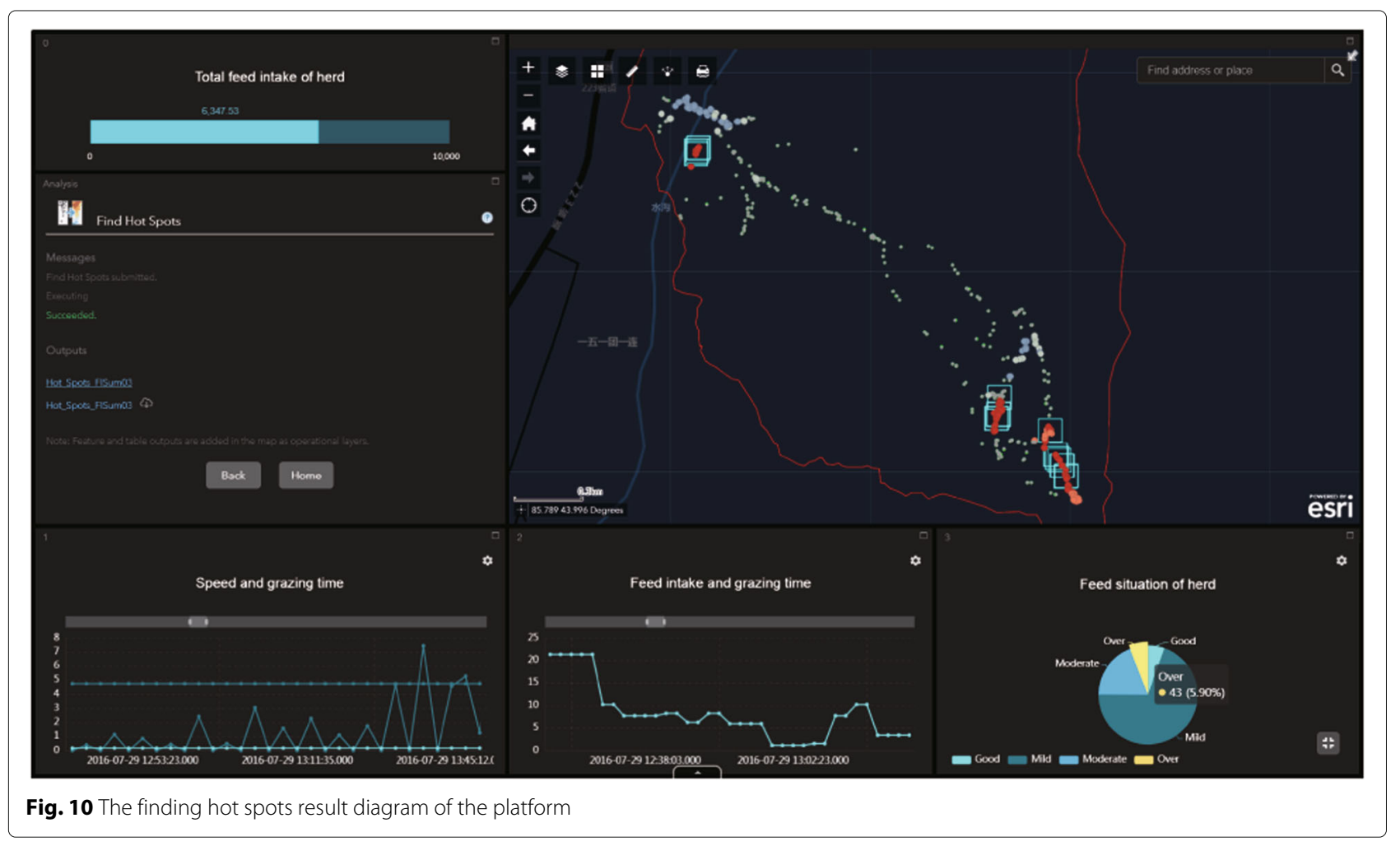


Users can select herd's trajectory points and analysis fields to conduct relevant hot spots analysis. Taking the trajectory points of the herd from July 28 to July 31, 2016 as an example, the hot spots result diagram was obtained, as shown in Fig. 10. The analysis field is feed intake of herd. It was found that the herd fed heavily in the starting point and the part farthest from the starting point by analyzing the hot point map of the feed intake. It was consistent with feeding characteristics of the herd. Interpolation analysis service is mainly applicable to large-scale pastures. Because the herd is large and relatively scattered. The feeding behavior of the whole herds can be predicted based on the trajectory of few herds. Taking feed intake of the herd from July 28 to July 31, 2016 as an example, the overall feed intake was predicted, and the result was shown in Fig. 11.

The third widget shows the relationship between walking speed of the herd and the grazing time. Li et al. [40] analyzed the walking speed range of the herd. Therefore, the maximum and minimum values of the walking speed range were used as reference lines in the line chart for users to analyze the walking speed of the herd. The walking speed of the herd obtained by this platform is slightly larger. This is related to the fact that Ziniquan pasture is located in the hilly area with relatively undulating terrain. The difference of vegetation, sheep species and monitoring time will also lead to the greater speed.
The fourth widget shows the relationship between feed intake of the herd and grazing time.

The fifth widget shows the pie chart of the feed situation of the herd.

Users can click to select the relational chart points or pie chart areas they are interested in. The main view will mark and show the selected trajectory points for users to view. Taking Fig. 11 as an example, click to select the over area of the pie chart, and the main view will mark and show the selected trajetory points.

\section{Discussion and conclusions}

Compared with previous studies of other researchers, the results of data analysis and visualization of this platform can better meet users' needs. The platform realized the functions of feed intake statistics, walking speed statistics, line chart analysis and pie chart analysis of spatial data related attributes, etc. Moreover, it also provides users with customized spatial analysis functions (such as buffer analysis, finding hot pots analysis and interpolation point analysis), which are not considered by other related visualization platforms. Traditional platform of analysis based on spatial data is that developers investigate users' needs and then design customized functions. However, the development cycle of visualization platform is very long. Once the developers investigate the users' needs improperly, they will face the loss of redevelopment. In addition, standard functions can only satisfy most users,

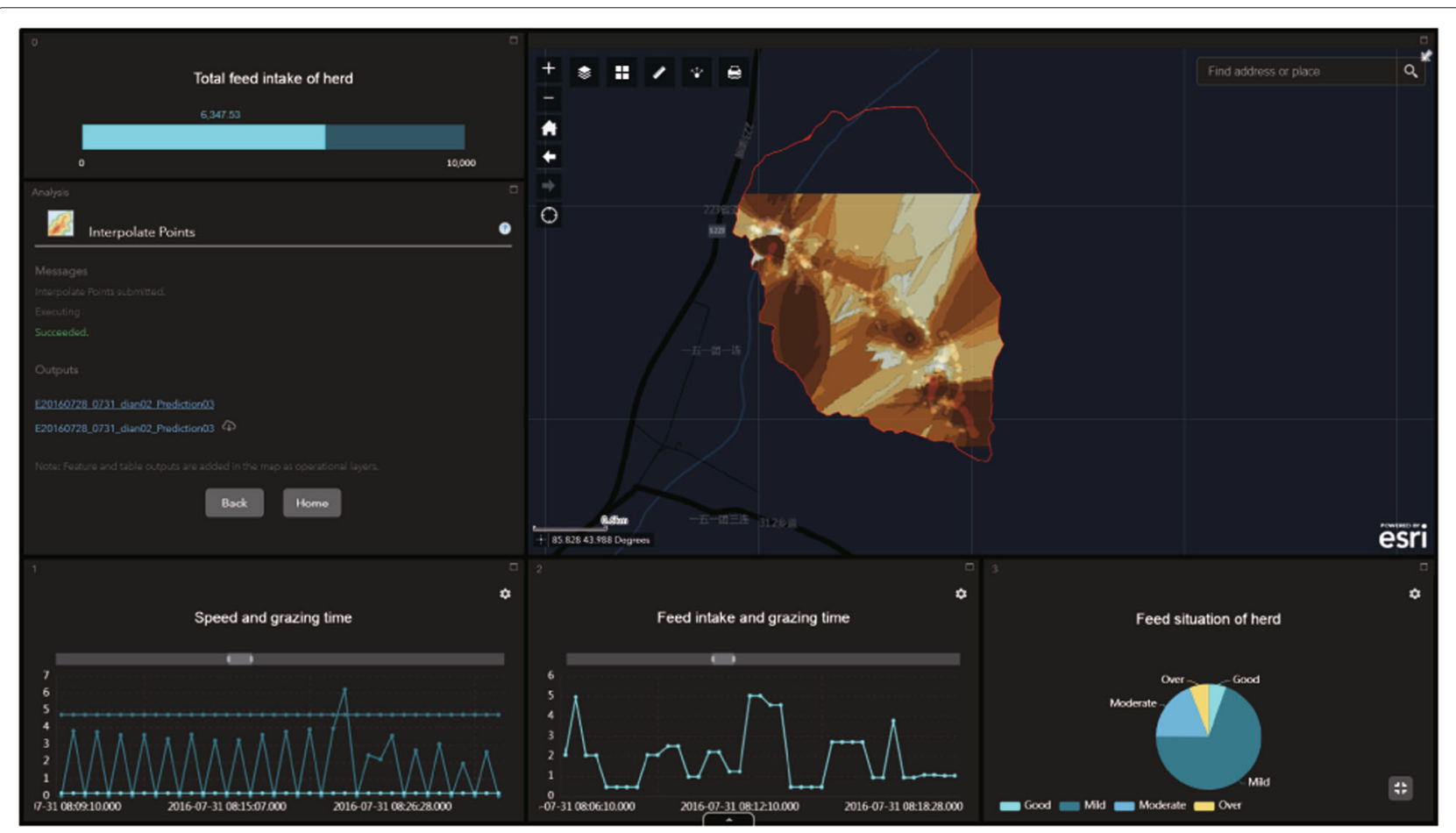

Fig. 11 The interpolation analysis result diagram of the platform 
but it is difficult to meet the needs of a few users. Thus, this platform took these pain points into account and was developed by Web AppBuilder for ArcGIS framework. The framework has some characteristics, such as fast development cycle and allowing users to customize analysis. Thereore, it can meet the special needs of some users effectively. Take the function of finding hot spots of spatial analysis as an example, different users may need to analyze different spatial data, while the find hotspot function enabled users to customize and select the spatial data and fields to analyze. It can allow some differences of data and not affect the analysis quality.

The platform can make ranchers and grassland livestock management departments grasp the grazing information of the herd in time. It can also provide the relevant researchers with a great understanding of the grazing behavior and habits of the herd. However, the platform can not monitor the grazing behavior of the herd in real time. For example, the walking speed and direction of the herd can't be measured in real time. In addition, the platform mainly relies on ArcGIS Online platform so that the display of results was slightly delayed. Therefore, it is necessary to add some functions such as grazing trajectory prediction, real-time monitoring of walking speed, and improve the loading speed of data in the future work.

\section{Abbreviations}

GP: Geoprocessing; GPS: Global Positioning System; GIS: Geographic Information System; GPRS: General Packet Radio Service; IOT: Internet Of Things; ECS: Elastic Compute Service

\section{Acknowledgements}

We highly appreciate the Yang Yonglin of the Xinjiang Academy of Agricultural Reclamation and the pastoralists of Ziniquan farm, who participated in the GPS tra-jectory data collection and shared their knowledge on herd. We are thankful to all the professional GIS technicians, graduate students and undergraduates who contrib-uted to the development of this platform. We are grateful for the thoughtful and con-structive comments of the reviewers that improved this manuscript in major ways.

\section{Authors' contributions}

$\mathrm{DL}$ is the main writer of this paper. He proposed the main idea and completed the development of the platform. CW contributed to the structuring, reviewing, and finalizing of the manuscript. QW, TY, WB and JW are the principal contributors in terms of writing and the discussion of results. All authors read and approved the final manuscript.

\section{Funding}

This work was supported by the National Key R \& D Program of China (Grant No.2017YFB0504203), the National Natural Science Foundation of China (Grant No. 41461088, and the XJCC XPCC Innovation Team of Geospatial Information Technology (Grant No. 2016AB001).

\section{Availability of data and materials}

The network behavior data used to support the findings of this study are available from the corresponding author upon request.

\section{Competing interests}

The authors declare that they have no competing interests.

\section{Author details}

${ }^{1}$ College of Information Science and Technology, Shihezi University, North Four Road, 832003 Shihezi, China. ${ }^{2}$ Geospatial Information Engineering Research Center, Xinjiang Production and Construction Corps, North Four
Road, 832003 Shihezi, China. ${ }^{3}$ School of Internet, Anhui University, Feixi Road, 230039 Hefei, China.

Received: 5 February 2020 Accepted: 23 June 2020

Published online: 23 October 2020

\section{References}

1. Zhao Q, Hou X, Duan j, Cheng R (2019) Production benefit trend analysis of grazing regime by sunite wether on mixed artificial pasture. Chin $J$ Grassl 41(03):130-135

2. Voormansik K, Jagdhuber T, Zalite K, Noorma M, Hajnsek I (2015) Observations of cutting practices in agricultural grasslands using polarimetric sar. IEEE J Sel Top Appl Earth Obs Remote Sens 9(4):1-15. https://doi.org/10.1109/JSTARS.2015.2503773

3. Chen Y, Lee G, Lee P, Oikawa T (2007) Model analysis of grazing effect on above-ground biomass and above-ground net primary production of a mongolian grassland ecosystem. J Hydrol 333(1):155-164. https://doi. org/10.1016/j.jhydrol.2006.07.019

4. Marquart A, Eldridge D, Travers S, Val J, Blaum N (2019) Large shrubs partly compensate negative effects of grazing on hydrological function in a semi-arid savanna. Basic Appl Ecol 38:58-68. https://doi.org/10.1016/j. baae.2019.06.003

5. Yu L, Chen Y, Sun W, Huang Y (2019) Effects of grazing exclusion on soil carbon dynamics in alpine grasslands of the tibetan plateau. Geoderma 353:133-143. https://doi.org/10.1016/j.geoderma.2019.06.036

6. Shen H, Zhu Y, Zhao X, Geng X, Gao S, Jingyun F (2016) Analysis on the current situation of grassland resources in china. Sci Bull 61(2):139-154

7. Liu J, Wang Z, Hao D, Li J, Han G (2018) Effect of heavy grazing on the organization ability of main plant species and functional groups in a desert steppe. Chin J Grassl 40(5):85-92

8. Yin GM, Qian HG, Wei ZJ, Liu JS, Yun-Hua LI, Tu BA (2009) Comparison of grazing sheep foraging behavior at different environment. Acta Agric Boreali Sin 24(4):205-208

9. Guo Q, Yin GM, Zhao HP, Huang YB, Sui YL (2011) Study on grazing behavior and feed intake of grazing sheep. Chin J Grassl 33(4):95-98

10. Wang S, Li Y (1997) A study on behaviour ecology of grazing sheep 3 influence of grazing time on grazing behaviour. Acta Prataculturalence 6(2):7-13

11. Wang HB, Ding XZ, Lang X, Liang CN, Yan P, Fa-Di LI (2012) Foraging behavior of oula sheep in summer pastures of maqu gannan. Acta Agrestia Sinica 20(3):583-588

12. Wang C, Wang W, Lu W, Wen C, Yin X, Qingzhan Z (2016) Distribution model of herd feed intake based on grazing time-space trajectory data. Trans Chin Soc Agric Eng 32(13):125-130

13. Benke KK, Sheth F, Betteridge K, Pettit CJ, Aurambout J-P (2015) Application of geovisual analytics to modelling the movements of ruminants in the rural landscape using satellite tracking data. Int J Digital Earth 8(7):579-593

14. Wu Q, Yang BJ, Zhang SL, Pei ZY (2001) 3s-based grassland information system for western china. Trans Chin Soc Agric Eng 17(5):142-145

15. Du Y, Peng H, Li B, Ling Q (2018) Research on regional attribute mining based on herd trajectory in massive pastoral areas. Appl Res Comput 35(4):1033-1036

16. Wang $X$ (2017) The effects of different grazing management system on livestock behavior. Inner Mongolia University, Hohhot

17. Pandey V, Kiker GA, Campbell KL (2009) Gps monitoring of cattle location near water features in south florida. Appl Eng Agric 25(4):551-562. https://doi.org/10.13031/2013.27465

18. Arnon A, Svoray T, Ungar ED (2013) The spatial dimension of pastoral herding: A case study from the northern negev. Isr J Ecol Evol 57(1):129-149. https://doi.org/10.1560/IJEE.57.1-2.129

19. Henkin Z, Ungar ED, Dolev A (2012) Foraging behaviour of beef cattle in the hilly terrain of a mediterranean grassland. Rangel J 34(2):163. https:// doi.org/10.1071/rj11096

20. Pérez J, Varga M, García J (2017) Monitoring lidia cattle with gps-gprs technology: a study on grazing behaviour and spatial distribution. Vet Mex 4(4). https://doi.org/10.21753/vmoa.4.4.405

21. Mysterud A, Rekdal Y, Loe LE (2014) Evaluation of landscape-level grazing capacity for domestic sheep in alpine rangelands. Vet Mex 67(2):132-144. https://doi.org/10.2111/REM-D-12-00177.1 
22. Liao C, Clark PE, Shibia M (2018) Spatiotemporal dynamics of cattle behavior and resource selection patterns on east african rangelands: evidence from gps-tracking. Int J Geogr Inf Syst 32(7):1523-1540. https:// doi.org/10.1080/13658816.2018.1424856

23. Akasbi Z, Oldeland J, Dengler J, Finckh M (2012) Social and ecological constraints on decision making by transhumant pastoralists: A case study from the moroccan atlas mountains. J Mt Sci 9(3):307-321. https://doi. org/10.1007/s11629-009-2320-8

24. Kawamura K, Akiyama T, Yokota HO, Tsutsumi M, Wang S (2005) Quantifying grazing intensities using geographic information systems and satellite remote sensing in the xilingol steppe region, inner mongolia, china. Agric Ecosyst Environ 107(1):83-93. https://doi.org/10.1016/j.agee. 2004.09.008

25. Chen J (2012) Design and implementation of satellite grazing system. East China Normal University, Shanghai

26. Cheng Z. L. (2015) Design and implementation of automatic track recognition system based on gps / gprs herd. Inner Mongolia University of Science and Technology, Hohhot

27. Xu X, Liu Q, Zhang X, Zhang J, Qi L, Dou W (2019) A blockchain-powered crowdsourcing method with privacy preservation in mobile environment. ieee transactions on computational social systems. IEEE Trans Comput Soc Syst 06:1407-1419. https://doi.org/10.1016/j.future.2019.01.012

28. Liu H, Kou H, Yan C (2019) Link prediction in paper citation network to construct paper correlation graph. EURASIP J Wirel Commun Netw 2019. https://doi.org/10.1186/s13638-019-1561-7

29. Li J, Cai T, Deng K, Wang X, Sellis T, Xia F (2020) Community-diversified influence maximization in social networks. Inf Syst 92:1-12

30. Xu X, Mo R, Dai F, Lin W, Wan S, Dou W (2020) Dynamic resource provisioning with fault tolerance for data-intensive meteorological workflows in cloud. IEEE Trans Ind Inform 16(9):6172-6181. https://doi. org/10.1109/TII.2019.2959258

31. Xu X, Shen B, Yin X, Khosravi M, Wu H, Qi L, Wan S (2020) Edge server quantification and placement for offloading social media services in industrial cognitive iov. IEEE Trans Ind Inform PP:1-1. https://doi.org/10. 1109/TII.2020.2987994

32. Chen Y, Zhang N, Zhang Y, Chen X, Wu W, Shen X (2019) Energy efficient dynamic offloading in mobile edge computing for internet of things. IEEE Trans Cloud Comput PP:1-1. https://doi.org/10.1109/TCC.2019.2898657

33. Xu X, Qingxiang L, Luo Y, Peng K, Zhang X, Meng S, Qi L (2019) A computation offloading method over big data for iot-enabled cloud-edge computing. Futur Gener Comput Syst 95:522-533. https:// doi.org/10.1016/j.future.2018.12.055

34. Zhou J, Sun J, Cong P, Liu Z, Wei T, Zhou X, Hu S (2019) Security-critical energy-aware task scheduling for heterogeneous real-time mpsocs in iot. IEEE Trans Serv Comput PP. https://doi.org/10.1109/TSC.2019.2963301

35. Zhou J, Hu X, Ma Y, Sun J, Wei T, Hu S (2019) Improving availability of multicore real-time systems suffering both permanent and transient faults. IEEE Trans Comput 68(12):1785-1801. https://doi.org/10.1109/TC. 2019.2935042

36. Zhou J, Sun J, Zhou X, Wei T, Chen M, Hu S, Hu X (2019) Resource management for improving soft-error and lifetime reliability of real-time mpsocs. IEEE Trans Comput Aided Des Integr Circ Syst 38(12):2215-2228. https://doi.org/10.1109/TCAD.2018.2883993

37. Qi L, Wang X, Xu X, Dou W, Li S (2020) Privacy-aware cross-platform service recommendation based on enhanced locality-sensitive hashing. IEEE Transactions on Network Science and Engineering PP:1-1. https:// doi.org/10.1109/TNSE.2020.2969489

38. Liu H, Kou H, Yan C, Qi L (2020) Keywords-driven and popularity-aware paper recommendation based on undirected paper citation graph. Complexity 2020:1-15. https://doi.org/10.1155/2020/2085638

39. Wang C, Jiang H, Lu W, Zhang Y, Sun S, Li D (2018) Evaluation model for natural grassland utilization based on spatio-temporal trajectory of grazing livestock. Trans Chin Soc Agric Mach 49(8):181-186

40. Li D, Qian Y, Wang C, Jiang H (2019) Estimation of walking speed of grazing sheep based on grazing spatio-temporal trajectory data. Chin J Grassl 41(5):152-159

\section{Publisher's Note}

Springer Nature remains neutral with regard to jurisdictional claims in published maps and institutional affiliations.

\section{Submit your manuscript to a SpringerOpen ${ }^{\circ}$ journal and benefit from:}

- Convenient online submission

- Rigorous peer review

- Open access: articles freely available online

- High visibility within the field

- Retaining the copyright to your article

Submit your next manuscript at $\boldsymbol{s p r i n g e r o p e n . c o m ~}$ 